\title{
On the Dramatic Spin-Up/Spin-Down Torque Reversals in BATSE Observations of Accretion Powered Pulsars
}

\author{
Robert W. Nelson ${ }^{1}$, Brian A. Vaughan, Lars Bildsten ${ }^{2}$, \\ Deepto Chakrabarty ${ }^{3}$, Danny T. Koh, and Thomas A. Prince \\ Space Radiation Laboratory, California Institute of Technology, \\ Pasadena, CA 91125 \\ Mark H. Finger ${ }^{4}$, Robert B. Wilson and Bradley C. Rubin \\ Space Science Laboratory, NASA/Marshall Space Flight Center, \\ Huntsville, AL 35812
}

\begin{abstract}
X-ray pulsars are the only accreting magnetic stars where rotation torques induced by accretion are large enough to be measured on short timescales $\sim$ days. They are thus unique laboratories for studying the interaction between an accretion disk and a stellar magnetosphere. We describe 5 years of continuous pulsar timing observations by the BATSE instrument on GRO which paint a strikingly different picture of pulsar spin behavior than understood from the previous 20 years of sparse observations. In particular, we find that more than half of the persistent pulsars we observe undergo dramatic torque reversals, switching suddenly between extended periods of steady spin-up and steady spin-down. Moreover, variations in pulsed flux are anticorrelated with torque in at least one system undergoing secular spin-down, GX1+4. This behavior contradicts standard accretion torque theory (Ghosh and Lamb 1979). A simple - albeit unconventional - hypothesis which naturally explains these observations is that the disks in these systems somehow alternate between epochs of prograde and retrograde rotation.
\end{abstract}

\section{Accretion-Powered Pulsars}

Accretion-powered pulsars are rotating, highly magnetized $\left(B \geq 10^{11} \mathrm{G}\right)$ neutron stars that accrete material from a stellar companion, either directly from a stellar wind, or by Roche-lobe overflow mediated by an accretion disk. Disks

\footnotetext{
${ }^{1}$ Theoretical Astrophysics, California Institute of Technology, Pasadena, CA 91104

${ }^{2}$ Current address: Department of Physics and Department of Astronomy, University of California, Berkeley, CA 94720

${ }^{3}$ Current address: Center for Space Research, Massachusetts Institute of Technology, Cambridge, MA 02139
}

${ }^{4}$ Universities Space Research Association 
may form in wind-fed systems if the material has sufficient angular momentum to circularize before reaching the neutron star magnetosphere. In the simplest picture of disk-fed pulsars, the strong magnetic field disrupts the disk and enforces the accreting plasma to corotate with the star at the magnetospheric radius, $r_{m}$, roughly where magnetic and fluid stresses balance. The plasma becomes entrained on magnetic field lines and falls to the stellar surface, releasing its gravitational potential energy as $\mathrm{x}$ - and gamma radiation at the magnetic poles. These rotating hot spots are the sources of the observed pulsed flux.

Assuming the specific angular momentum of material captured from the inner accretion disk is carried onto the neutron star, the pulsar will experience a spin-up torque (Pringle and Rees 1972; Rappaport and Joss 1977)

$$
N=\dot{M} \sqrt{G M r_{m}} .
$$

Using standard accretion theory, the neutron star will spin up at a rate

$$
\dot{f}=N /(2 \pi I) \simeq 4 \times 10^{-12} L_{37}^{6 / 7} \mu_{30}^{2 / 7} I_{45}^{-1} \mathrm{~Hz} / \mathrm{s},
$$

where $L=L_{37} 10^{37} \mathrm{ergs} \mathrm{s}^{-1}$ is the accretion luminosity, $\mu=\mu_{30} 10^{30} \mathrm{G} \mathrm{cm}^{3}$ is the neutron star magnetic moment, and $I=I_{45} 10^{45} \mathrm{gm} \mathrm{cm}^{2}$ is its moment of inertia. Early observations indicated that many pulsars were spinning up - on average - on a timescale $t_{s}=-f / \dot{f} \sim 10^{4} \mathrm{yrs}$, consistent with equation (2) (Rappaport and Joss 1977). This confirmed the hypothesis that $\mathrm{x}$-ray pulsars must be neutron stars with small moments of inertia, but large magnetospheric radii.

Accretion torques also act on other types of magnetic stars accreting from disks, such as magnetic CVs and T Tauri stars (Warner 1990; Konigl 1991). However, because a neutron star has a relatively small moment of inertia, $\sim 10^{-6}$ times that of a white dwarf and $\sim 10^{-10}$ that of a T Tauri star, it responds much more rapidly to accretion torque, typically $\Delta f \sim 10^{-7} \mathrm{~Hz}$ per day. Such changes can be measured in one day with current instruments. Consequently, accretionpowered pulsars are the only magnetic stars where the disk-magnetospheric interaction can be studied dynamically.

\section{The Model of Ghosh and Lamb}

Already in the 1970s two exceptions suggested that the simple torque estimate in equation (1) was sometimes inadequate: Her X-1 and Cen X-3 were known to be spinning up on a timescale much longer than predicted by equation (2) (Elsner and Lamb 1977; Ghosh \& Lamb 1979). Moreover, both systems sometimes underwent short episodes of spin-down. How could a star capturing material from a disk with the same sense of rotation actually lose angular momentum while continuing to accrete?

Ghosh and Lamb (1979; hereafter GL) argued that these stars must rotate near the equilibrium frequency where the inner edge of the Keplerian accretion disk corotates with the star, $\Omega_{*} \simeq \Omega_{K}\left(r_{m}\right)=\left(G M / r_{m}^{3}\right)^{1 / 2}$. Making a number of simplifying assumptions about the nature of the complex boundary layer between the disk and the magnetosphere, they found that additional negative 


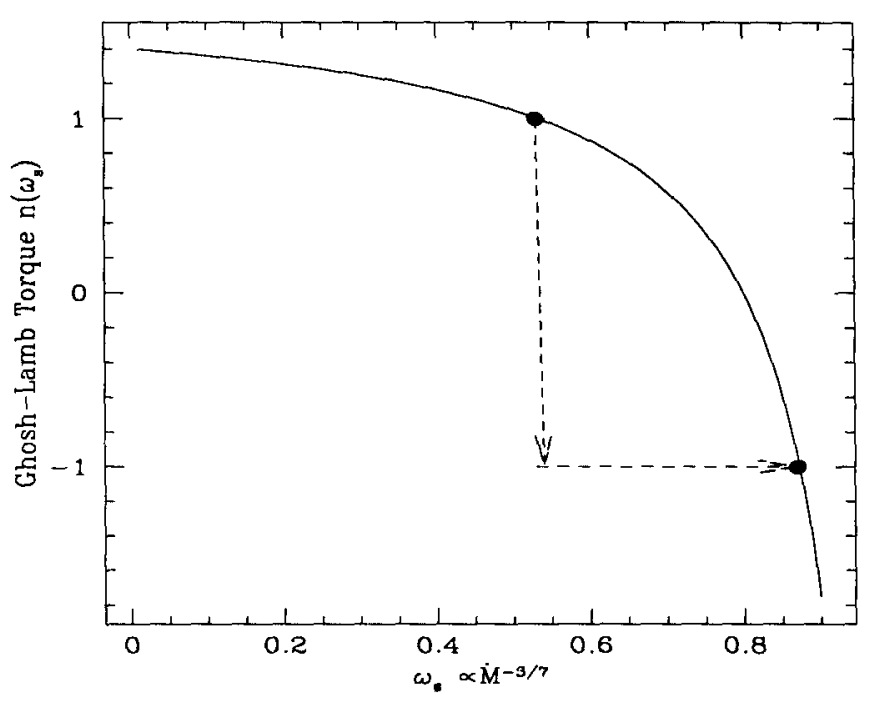

Figure 1. The Ghosh-Lamb dimensionless accretion torque is a smooth function of mass accretion rate through $\omega_{s}=\Omega_{*} / \Omega_{K}\left(r_{m}\right) \propto$ $\dot{M}^{-3 / 7}$. Sudden changes in spin-up torque, as occured in $4 \mathrm{U} 1626-67$ (figure 3), require sudden step-function-like changes in mass accretion rate.

torques must act on the star; magnetic field lines which thread the disk beyond the corotation radius - where the disk rotates more slowly than the star - are swept back in a trailing spiral and transport angular momentum outward. Stars sufficiently close to equilibrium can spin-down while continuing to accrete.

The total GL torque can be written as a modified form of equation (1),

$$
N_{G L}=n\left(\omega_{s}\right) \dot{M} \sqrt{G M r_{m}}
$$

where $n\left(\omega_{s}\right)$ is a universal dimensionless function of the "fastness parameter", $\omega_{s}=\Omega_{*} / \Omega_{K}\left(r_{m}\right)$. Although its exact functional form has been somewhat controversial (e.g. Campbell 1987; Wang 1987; 1996), for our discussion it is only important that $n\left(\omega_{s}\right)$ is a smooth monotonic function which decreases with $\omega_{s} \propto \dot{M}^{-3 / 7}$, and vanishes at some critical fastness parameter, $\omega_{c} \lesssim 1$. An approximate version of $n\left(\omega_{s}\right)$ with $\omega_{c}=0.8$ is shown in figure 1 . Stars accreting at constant rates will evolve towards the equilibrium spin frequency, $\Omega_{e q}=\omega_{c} \Omega_{K}\left(r_{m}\right)$, where the total torque vanishes. Observed fluctuations in torque mainly reflect the dependence of torque on the mass accretion rate, through $r_{m} \propto \dot{M}^{-2 / 7}$. In particular, transitions from spin-up to spin-down can occur if $\dot{M}$ drops suddenly and the magnetosphere moves outwards, sufficiently close to the corotation radius where the negative magnetic torques become large.

This picture of a magnetically threaded disk giving rise to spin-down torques in near-equilibrium stars has generally been accepted as the correct description of accretion torques acting in X-ray pulsars. Moreover, the theory has been carried over directly to similar situations thought to occur in cataclysmic variables and 
T-Tauri stars (Warner 1990; Konigl 1991; Ghosh 1995). Yet there has been very little corroborating evidence to support or reject the GL model, mainly because timing measurements in X-ray astronomy have been too sparse, and flux measurements too imprecise, to test the predicted correlations between torque and luminosity. As we discuss below, in at least one system undergoing secular spin-down the observed correlation is the opposite of that predicted by GL.

\section{Observations with BATSE}

Prior to 1991, the spin periods of accreting pulsars were typically measured only once or twice per year due to tightly constrained observing schedules of orbiting X-ray telescopes. Since the launch of BATSE in April 1991, we have been monitoring pulse frequencies for roughly half of the known X-ray pulsars. BATSE is the first instrument capable of compiling a continuous, long-term history of pulse frequencies for the majority of persistent X-ray pulsars.

These observations use the 8 Large-Area Detectors (LADs) that face out from the corners of the CGRO spacecraft with overlapping, $2 \pi$ SR fields of view. The detectors are non-imaging and sensitive from $20 \sim 2000 \mathrm{keV}$, with an effective area of $\sim 1500 \mathrm{~cm}^{2}$ at $40 \mathrm{keV}$ and an energy resolution of $35 \%$ FWHM. For scientific analysis we primarily use DISCLA data, sampled every $1.024 \mathrm{~s}$ in 4 energy channels, and CONT data, sampled every $2.048 \mathrm{~s}$ in 16 energy channels. The large, highly-variable background is subtracted by fitting an approximate model prior to scientific analysis. Rates from detectors facing the pulsar are summed and then epoch folded for a daily measurement of spin frequency, pulse profile, and pulsed flux. The large, highly variable background and restricted energy range of the LADs mean that we can only measure the pulsed component of the flux, and only above $20 \mathrm{keV}$.

Using BATSE, we have compiled continuous histories containing 150 5000 frequency and pulsed flux measurements spanning the past 5 years for each of the persistent X-ray pulsars Her X-1, Cen X-3, 4U 1626-67, OAO 1657-415, GX $1+4$, Vela X-1, 4U 1538-52, and GX 301-2. The typical pulsar is sampled on a daily basis. See Vaughan et al. (1997) for an overview of BATSE pulsed-source observations.

One example where BATSE observations reveal a strikingly different picture of pulsar spin behavior is the frequency history of the $4.8 \mathrm{~s}$ pulsar Cen X-3, shown in figure 2. Prior to BATSE, the long-term frequency history (figure 2 a) has been described as secular spin-up at $\dot{f}=8 \times 10^{-13} \mathrm{~Hz} \mathrm{~s}^{-1}$ - a factor of $\sim 5$ slower than predicted by eq. 2 - superposed with wavy fluctuations and short episodes of spin down. This behavior suggested Cen X-3 rotates near its equilibrium spin period (Ghosh and Lamb 1979). In contrast, the more frequently sampled BATSE data (figure $2 \mathrm{~b}$ ) show that Cen X-3 exhibits $10 \sim 100 \mathrm{~d}$ intervals of steady spin up and spin down at a much larger rate consistent with equation 2 . Transitions between spin up and spin down occur on a time scale more rapid than BATSE can resolve $\leqslant 1 \mathrm{~d}$. More significantly, the apparent small torque inferred from the pre-BATSE data is an illusion, a consequence of the frequent transitions between spin up and spin down. 

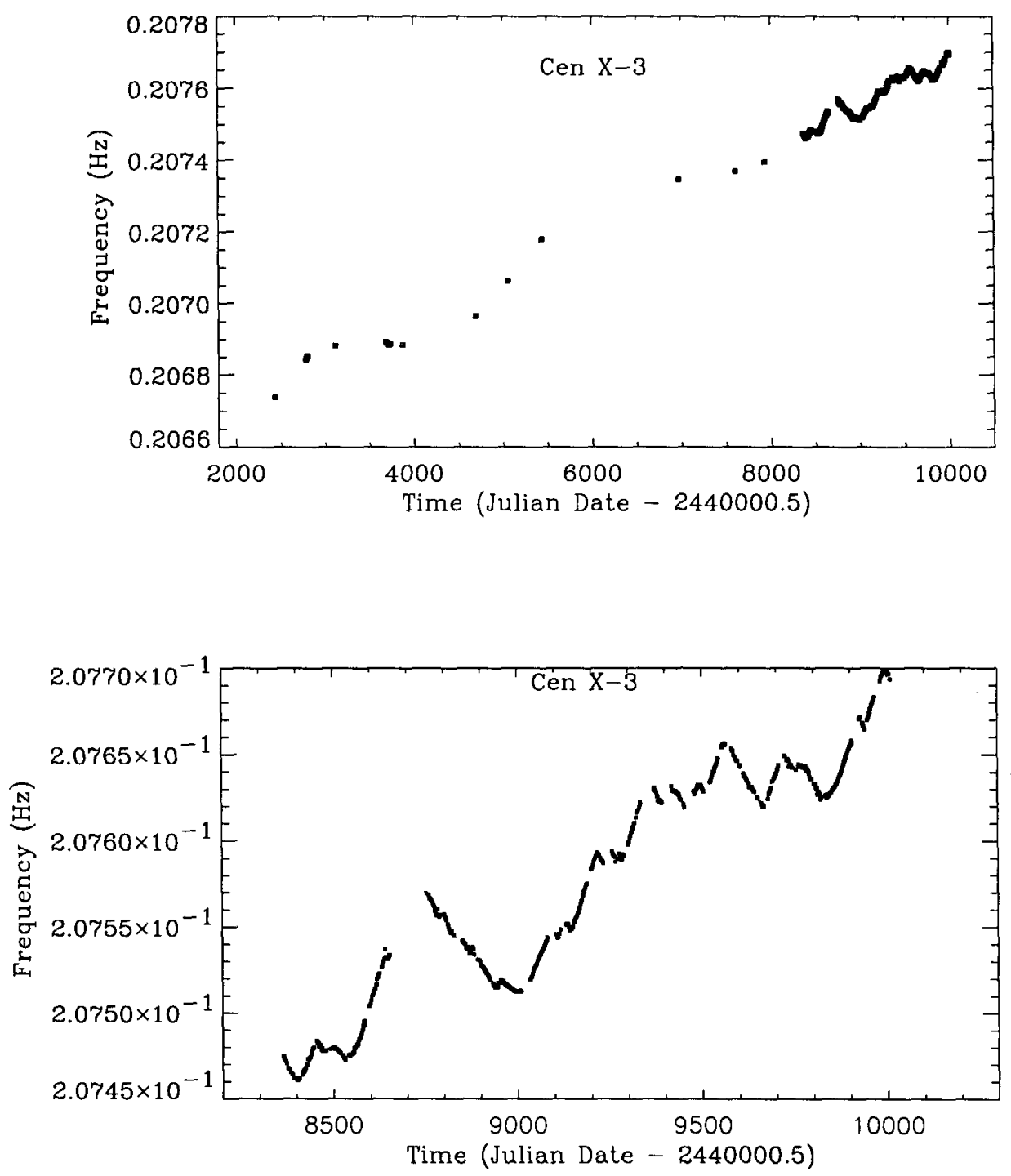

Figure 2. (a) The long term frequency history of Cen X-3. Prior to the BATSE observations, its behavior was described as a slow secular spin-up superposed with wavy fluctuations and short episodes of spindown. (b) High resolution BATSE frequency history of Cen X-3. The torque appears to be bimodal, alternating between spin-up and spindown, with an instantaneous magnitude much larger than its average value. 


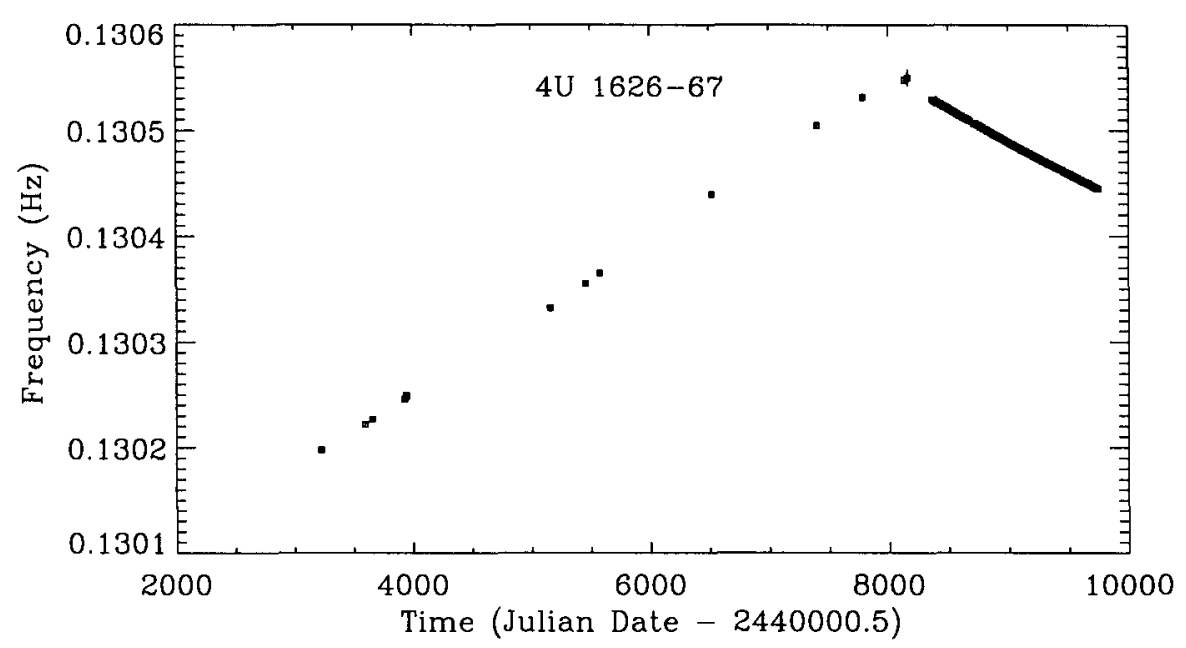

Figure 3. The long term spin frequency of 1626-67 (from Chakrabarty et al. 1997a). The magnitude of the spin-down torque is within $15 \%$ of the spin-up value

\section{Dramatic Torque Reversals Seen in X-ray Pulsars}

The bimodal torque behavior seen in Cen X-3 has been confirmed quantitatively (Finger, Wilson \& Fishman 1994). Interestingly, this behavior appears to be quite common: 5 out of the 8 persistent pulsars observed by BATSE show torque reversals between steady spin-up and steady spin-down. Of the remaining three, two (GX 301-2, Vela X-1) are wind-fed pulsars and Her X-1 is sampled infrequently at 35 day intervals so that we cannot measure its torque on short timescales.

One of the most dramatic torque transitions took place in the $7.6 \mathrm{~s}$ pulsar $4 \mathrm{U}$ 1626-67 around 1991, and is shown in figure 3 (Chakrabarty et al. 1997a). After two decades of the smoothest spin-up observed in any accreting pulsar, BATSE found that 4 U1626-67 was smoothly spinning down. Most surprisingly, the final torque is nearly equal in magnitude $\left(7 \times 10^{-13} \mathrm{~Hz} \mathrm{~s}^{-1}\right)$ but opposite in sign to the initial spin-up torque. A similar transition to spin down was observed in the 120 s pulsar GX $1+4$ in 1988 (Makishima et al. 1988) after more than a decade of steady spin up (figure 4). Again, the spin down rate $\left(3.7 \times 10^{-12} \mathrm{~Hz} \mathrm{~s}^{-1}\right)$ is close in magnitude to the spin up rate. In the $38 \mathrm{~s}$ disk-fed pulsar OAO 1657-415 both the duration and strength of torque episodes are very close to those seen in Cen X-3 (Chakrabarty et al. 1993; Vaughan et al. 1997). Finally, the $530 \mathrm{~s}$ pulsar $4 \mathrm{U} 1538-52$ is found to be spinning up at $2 \times 10^{-14} \mathrm{~Hz} \mathrm{~s}^{-1}$ after a decade of spin-down at a similar rate (Rubin et al. 1997), although the sparseness of pre-BATSE measurements cannot preclude other torque reversals.

The well-studied pulsars $4 \mathrm{U} 1626-67$ and GX 1+4, with long episodes of steady spin-up and spin-down, show secular and stochastic variations in torque 


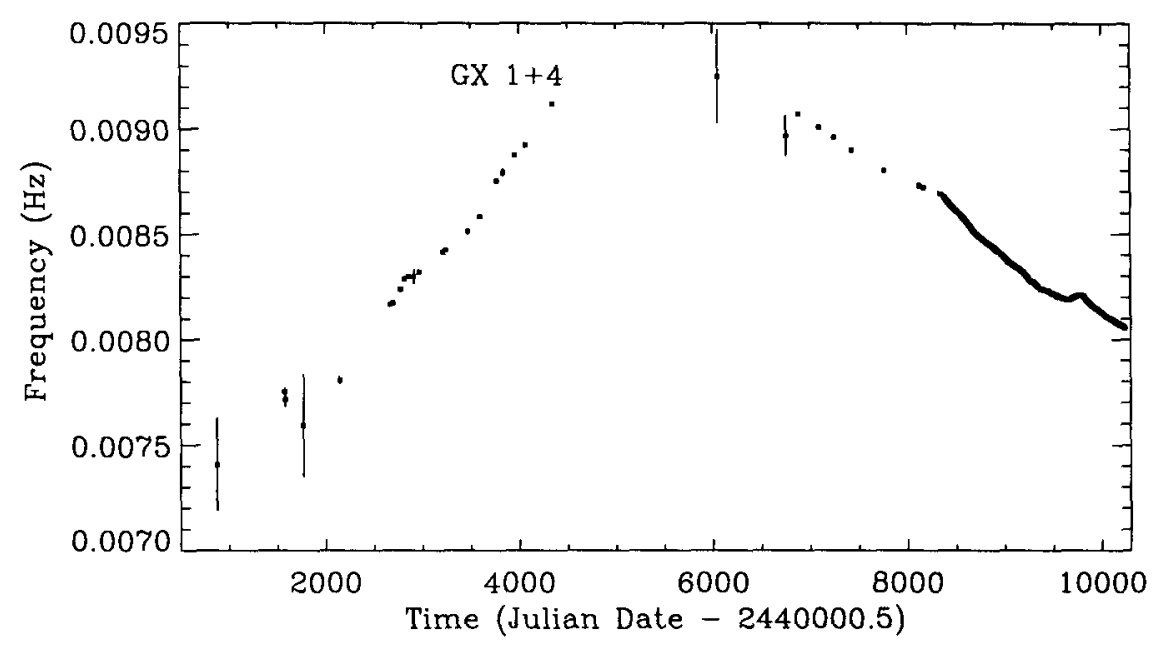

Figure 4. GX 1+4 long term frequency history (Chakrabarty et al. 1997b).

and luminosity in addition to torque reversals. The rate of spin-down is changing at $|\ddot{f} / \dot{f}| \approx 12$ and 10 years respectively. Moreover, in both cases the flux and the magnitude of the accretion torque decreased somewhat in going from spinup to spin-down, (Chakrabarty 1997a,b). Most interestingly, BATSE observed a number of bright hard X-ray flares in GX $1+4$ of 10-30 day duration accompanied by episodes of enhanced spin-down (figure 5), and a reversal to spin-up lasting approximately 200 days in 1995, accompanied by persistently high luminosity. As discussed below, this behavior is inconsistent with the GL model of accretion torques.

\section{Difficulties with Ghosh and Lamb Model}

Given the sparse observations available through the 1980s, the GL model provided a plausible mechamism for spin-down. However, it cannot explain many of the BATSE observations.

1. The observed torque appears to be bimodal, switching rapidly between two possible states. In the GL model, the torque should smoothly track changes in the mass accretion rate (figure 1). Consequently, sudden changes in torque require step-function-like changes in $\dot{M}$.

2. The torque always alternates between states of opposite signs (see figure $2 \mathrm{~b}$ ), and comparable magnitudes. In the GL model this requires the two values of $\dot{M}$ to be finely tuned. Why do we never see Cen X-3 jump between torque states with the same sign? Why should a system like $4 \mathrm{U} 1626$ - 


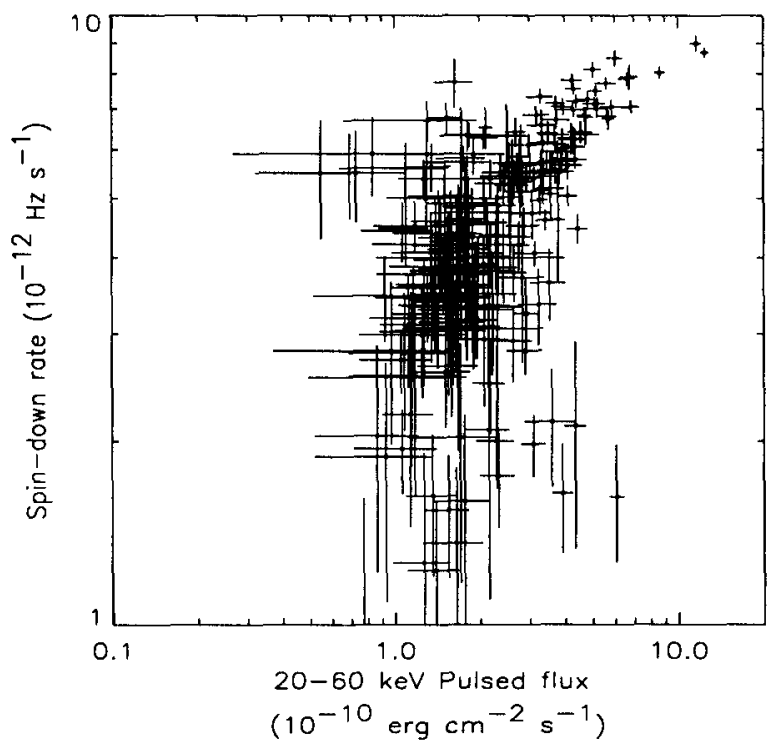

Figure 5. The torque is anticorrelated with pulsed luminosity in GX 1+4. This is opposite the trend predicted by Ghosh and Lamb (1979), but is exactly the expected behavior if GX $1+4$ is accreting from a disk rotating with the opposite sense.

67 undergo such a a dramatic and finely tuned transition (Chakrabarty 1997a)?.

3. In its extended spin-down state, during short-term ( $\sim 10$ day) flares, GX $1+4$ actually spins down faster (figure 5 ). That is, the torque is anticorrelated with luminosity (Chakrabarty et al. 1997b). This is opposite the prediction of GL; at higher accretion rates the magnetosphere moves inward, reducing the magnetic spin-down torques while increasing the material spin-up torques.

4. To be near equilibrium and spinning down, the GL model requires the slowly rotating pulsar $\mathrm{GX} 1+4\left(P_{p} \simeq 120 \mathrm{~s}\right)$ to have a large magnetospheric radius, $r_{m} \sim 4 \times 10^{9} \mathrm{~cm}$, and ultra-strong dipole field strength, $B_{d} \sim 10^{14}$ G. This would be the largest known magnetic field of any neutron star.

5. The natural dynamical and viscous timescales at the disk-magnetosphere boundary ranges from seconds to hours. Why should systems like GX $1+4$ and $4 \mathrm{U} 1626-67$ be so stable for years between torque transitions?

\section{Retrograde Disks}

When spin-down was first detected in GX 1+4, Makishima et al. (1988) suggested that a new disk had formed with a reversed sense of direction. This seemed implausible for any system undergoing Roche-lobe overflow, since then 
the accreting material should always have the same sense of angular momentum as the orbit. Instead, they suggested that GX $1+4$ was accreting from a dense, subsonic wind from its $\mathrm{M}$ giant companion; transient formation of alternating prograde and retrograde disks are known to occur in wind-fed systems, albeit on short timscales (Fryxell and Taam 1988). This idea was not taken seriously at the time (White 1988), however, since a low velocity wind carries a large specific angular momentum, $l \propto v^{-4}$, with the same sense as the orbit.

Nevertheless, this hypothesis - that alternating episodes of prograde and retrograde disks do occur - resolves the difficulties listed in the previous section.

1. Disk reversals naturally lead to two-state torque behavior, since the material must circulate one way or the other.

2. The two torques must always be of opposite sign, and of comparable magnitude for similar accretion rates (eq. 1).

3. The anticorrelation between torque and luminosity observed in GX $1+4$ is expected, since material from a retrograde disk carries negative angular momentum relative to the neutron star rotation.

4. If spin-down is caused by the formation of a retrograde disk, GX $1+4$ need not be in equilibrium with $\Omega_{*} \simeq \Omega\left(r_{m}\right)$, or have an ultra-strong magnetic field.

5. The only natural timescale comparable to the observed intervals between torque reversals is the global disk viscous time $\sim$ weeks to years for a standard $(\alpha \sim 0.01-0.1)$ Shakura-Sunyaev disk. One can imagine a cycle where disks form, accrete all of their material, and then form with the opposite sense of rotation.

At present there is no physical explanation for the formation of retrograde disks, however, or their long-term stability. It remains to be seen whether further observations remain consistent with this hypothesis.

\section{References}

Campbell, C. G. MNRAS, 229, 405

Chakrabarty, D. et al. 1997a, ApJ, 474, in press

Chakrabarty, D. et al. 1997b, ApJ, in preparation

Elsner, R. F., \& Lamb, F. K. 1977, ApJ, 215, 897

Finger, M. H., Wilson, R. B. \& Fishman, G. J. 1994, in Compton Gamma-Ray Observatory: AIP Conference Proceedings 280, NY, AIP Press, 304

Fryxell, B. A., \& Taam, R. E. 1988, ApJ, 335, 862

Ghosh, P 1995, MNRAS, 272, 763

Ghosh, P, \& Lamb, F. K. 1979, ApJ, 234, 296

Konigl, A 1991, ApJ, 370, L39

Makishima, K. et. al. 1988 Nature, 333, 746 
Pringle, J. E. \& Rees, M. J. 1972 A\&A, 21, 1

Rappaport, S. \& Joss, P. 1977 Nature, 266, 683

Rubin et al. 1997, ApJ, in preparation

Wang, Y.-M. 1987, A\&A, 183, 257

Wang, Y.-M. 1995, ApJ, 449, L153

Warner, B 1990, Ap\&SS, 164, 79

White, N. 1988, Nature, 266, p 708

Vaughan et al. 1997, ApJ, in preparation 